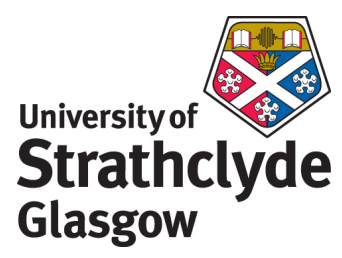

Scheurich, F. and Fletcher, T.M. and Brown, R.E. (2010) The influence of blade curvature and helical blade twist on the performance of a vertical-axis wind turbine. In: 29th ASME Wind Energy Symposium, 4-7 January 2010, Orlando, Florida.

http://strathprints.strath.ac.uk/27341/

Strathprints is designed to allow users to access the research output of the University of Strathclyde. Copyright $(C$ and Moral Rights for the papers on this site are retained by the individual authors and/or other copyright owners. You may not engage in further distribution of the material for any profitmaking activities or any commercial gain. You may freely distribute both the url (http://strathprints.strath.ac.uk) and the content of this paper for research or study, educational, or not-for-profit purposes without prior permission or charge. You may freely distribute the url (http://strathprints.strath.ac.uk) of the Strathprints website.

Any correspondence concerning this service should be sent to The Strathprints Administrator: eprints@cis.strath.ac.uk 


\title{
The Influence of Blade Curvature and Helical Blade Twist on the Performance of a Vertical-Axis Wind Turbine
}

Frank Scheurich*

\author{
Timothy M. Fletcher ${ }^{\dagger}$ \\ Rotor Aeromechanics Laboratory \\ Department of Aerospace Engineering \\ University of Glasgow \\ Glasgow, G12 8QQ, United Kingdom
}

Richard E. Brown $\ddagger$

\begin{abstract}
Accurate aerodynamic modeling of vertical-axis wind turbines poses a significant challenge, but is essential if the performance of such turbines is to be predicted reliably. The rotation of the turbine induces large variations in the angle of attack of its blades that can manifest as dynamic stall. In addition, interactions between the blades of the turbine and the wake that they produce can exacerbate dynamic stall and result in impulsive changes to the aerodynamic loading on the blades. The Vorticity Transport Model has been used to simulate the aerodynamic performance and wake dynamics of vertical-axis wind turbines with straight-bladed, curved-bladed and helically twisted configuration. It is known that vertical-axis wind turbines with either straight or curved blades deliver torque to their shaft that fluctuates at the blade passage frequency of the rotor. In contrast, a rotor with helically twisted blades delivers a relatively steady torque to the shaft. In the present paper, the interactions between helically twisted blades and the vortices within their wake are shown to result in localized perturbations to the aerodynamic loading on the rotor that can disrupt the otherwise relatively smooth power output that is predicted by simplistic aerodynamic tools that do not model the wake to sufficient fidelity. Furthermore, verticalaxis wind turbines with curved blades are shown to be somewhat more susceptible to local dynamic stall than turbines with straight blades.
\end{abstract}

\section{Nomenclature}

Presented at the 48th AIAA Aerospace Sciences Meeting Including the New Horizons Forum and Aerospace Exposition, Orlando, Florida, USA, 4-7th January 2010. Copyright (c) 2010 by Frank Scheurich, Timothy M. Fletcher and Richard E. Brown. Published by the American Institute of Aeronautics and Astronautics, Inc., with permission. 


$\begin{array}{ll}P & \text { power } \\ Q^{*} & \text { sectional non-dimensional torque, } F_{t}^{*} r / R \\ r=r(b) & \text { sectional radius } \\ R & \text { radius of the rotor at blade mid-span } \\ R e & \text { blade (rotational) Reynolds number, } R e=\Omega R c / \nu \\ S & \text { vorticity source } \\ u & \text { flow velocity } \\ u_{b} & \text { flow velocity relative to the blade } \\ V_{\infty} & \text { wind speed } \\ \alpha & \text { angle of attack } \\ \lambda & \text { reference tip speed ratio, } \Omega R / V_{\infty} \\ \nu & \text { kinematic viscosity } \\ \psi & \text { azimuth angle } \\ \rho & \text { density } \\ \omega & \text { vorticity } \\ \omega_{b} & \text { bound vorticity } \\ \Omega & \text { angular velocity of the rotor }\end{array}$

\section{Introduction}

The number of wind turbines deployed around the globe has increased significantly in recent years in an attempt to curb emissions of carbon dioxide and other pollutants. The wind turbines that are currently operating in wind farms feature, almost exclusively, a three-bladed rotor with a horizontal-axis configuration. In recent years, however, there has been a resurgence in the development of both large-scale and small-scale vertical-axis wind turbines. The development of large-scale vertical-axis wind turbines is mainly a response to a plateau in the improvement of the aerodynamic performance of horizontal-axis wind turbines, whereas the research into small-scale vertical-axis wind turbines is motivated by a perceived future demand for decentralized electricity generation within cities and rural communities.

Vertical-axis wind turbines are, by design, insensitive to the wind direction if the wind is perpendicular to the axis of rotation, and therefore do not require a yaw control system. Horizontal-axis wind turbines, in contrast, have to be rotated in order to track changes in wind direction. Several authors, including Mertens, ${ }^{1}$ have suggested that vertical-axis wind turbines offer distinct advantages over those with horizontal-axis design when operating in conditions that are typical of urban environments.

A vertical-axis wind turbine is called a Darrieus turbine when it is driven by aerodynamic lift. The concept was patented in the United States by Georges Darrieus ${ }^{2}$ in 1931 . The patent includes both straightand curved-bladed configurations in which the blades are supported using plates located at the top and the bottom of the turbine to provide structural strength. By curving the blades to yield the so-called troposkien shape, which is equivalent to the shape of a spinning rope that is constrained at its ends, the high bending moments that are encountered on straight-bladed turbines can, to some extent, be alleviated. Both straightbladed and curved-bladed vertical-axis wind turbines suffer, however, from a marked azimuthal variation in the loading on their blades. These variations in loading manifest as oscillations in the torque and power that is output from the turbine. The oscillating torque that is developed by straight- and curved-bladed turbines contributes significantly to the vibration that is transmitted to the tower and the foundations of the system. In addition, the oscillations in the loading on the blades can lead to increased fatigue of the rotor structure. For these reasons, several commercial vertical-axis wind turbines feature a design that incorporates blades that are twisted helically around the rotational axis of the turbine. The torque, and consequently the power output, of vertical-axis wind turbines with helically twisted blades is relatively steady, thus alleviating the stress on the turbine system, reducing the vibration transmitted into its supporting structure, and thereby increasing the design life of the turbine.

The accurate aerodynamic simulation of vertical-axis wind turbines poses a significant challenge. During operation, the angle of attack of the blades varies cyclically with azimuth. Dynamic stall can occur at low tip speed ratios when the angle of attack of the blades transiently exceeds the static stall angle of their airfoil sections. In addition, the interactions between the blades of the turbine and the vortices in its wake create an impulsive component to the aerodynamic loading on the blades. These interactions are 
notoriously difficult to predict accurately in terms of their position and strength. Indeed, Klimas ${ }^{3}$ considered the interaction between the blades of the turbine and the wake produced by the rotor to be one of the most critical problems in the numerical modeling of the aerodynamics of vertical-axis wind turbines. In the present paper, a computational model is used to compare the aerodynamic performance of a turbine with straight blades to that of a second turbine with curved blades, and to that of a third turbine with blades that are helically twisted around the rotational axis. The results presented in this paper indicate that the model that is used for the aerodynamic simulation of the different vertical-axis wind turbine configurations is capable of capturing the interactions between the blades of the turbine and the wake that is developed by the rotor, thus allowing a detailed analysis of the influence of the blade-wake interactions on the distribution of the aerodynamic loading along the blades of the turbine.

\section{Computational Aerodynamics}

The aerodynamic performance and wake dynamics of three vertical-axis wind turbine configurations have been simulated using the Vorticity Transport Model (VTM) developed by Brown, ${ }^{4}$ and extended by Brown and Line. ${ }^{5}$ The VTM enables the simulation of wind turbine aerodynamics and performance by providing a high-fidelity representation of the dynamics of the wake that is generated by the turbine rotor. In contrast to more conventional computational fluid dynamics techniques that are based on the pressure-velocity-density formulation of the Navier-Stokes equations, the VTM is based on the vorticity-velocity form of the unsteady Navier-Stokes equations. After making the physically realistic assumption of incompressibility within the wake, the Navier-Stokes equations can be written in the vorticity-velocity form

$$
\frac{\partial}{\partial t} \omega+u \cdot \nabla \omega-\omega \cdot \nabla u=S+\nu \nabla^{2} \omega
$$

The advection, stretching, and diffusion terms within the vorticity transport equation describe the changes in the vorticity field, $\omega$, with time at any point in space, as a function of the velocity field, $u$, and the viscosity, $\nu$. The physical condition that vorticity may neither be created nor destroyed within the flow, and thus may only be created at the solid surfaces that are immersed within the fluid, is accounted for using the vorticity source term, $S$. The vorticity source term is determined as the sum of the temporal and spatial variations in the bound vorticity, $\omega_{b}$, on the turbine blades, so that

$$
S=-\frac{d}{d t} \omega_{b}+u_{b} \nabla \cdot \omega_{b}
$$

In the VTM, equation (1) is discretized in finite-volume form using a structured Cartesian mesh within the fluid domain surrounding the turbine rotor, and then advanced through time using an operator-splitting technique. The numerical diffusion of vorticity within the flow field surrounding the wind turbine is kept at a very low level by using a Riemann problem technique based on the Weighted Average Flux method developed by Toro $^{6}$ to advance the vorticity convection term in equation (1) through time. This approach permits many rotor revolutions to be captured without significant spatial smearing of the wake structure, in contrast to the performance of those techniques that are based on the pressure-velocity-density formulation of the Navier-Stokes equations. Dissipation of the wake does still occur, however, through the proper physical process of natural vortical instability.

The bound vorticity distribution on the blades of the rotor is modeled using an extension of lifting-line theory. The lifting-line approach has been appropriately modified by the use of two-dimensional experimental data in order to represent the real performance of any given airfoil. The effect of dynamic stall on the aerodynamic performance of an airfoil is accounted for by using a Leishman-Beddoes-type semi-empirical dynamic stall model. ${ }^{7}$ The Leishman-Beddoes model was originally developed to simulate the effect of dynamic stall on the blades of a helicopter. Gupta and Leishman ${ }^{8}$ demonstrated, however, that a modified version of the original Leishman-Beddoes model can be used to represent the dynamic stall of airfoils of the type that are typically used for horizontal-axis wind turbines. The Leishman-Beddoes-type dynamic stall model that is implemented in the VTM is based on Beddoes's third generation dynamic stall model, ${ }^{9}$ but has been modified, as suggested by Niven and Galbraith, ${ }^{10}$ to account for vortex inception at low Mach numbers.

The VTM has been validated against experimental data ${ }^{11}$ for a horizontal-axis wind turbine and compared with equivalent predictions made using RANS solvers. ${ }^{12}$ The VTM has also been used successfully to investigate the aerodynamic interaction between the horizontal-axis wind turbines within a wind farm. ${ }^{13}$ 


\section{Turbine Model}

The geometry of each of the three vertical-axis wind turbines that are investigated in this paper is illustrated in figure 1. The blades of each turbine are separated by $120^{\circ}$ azimuth. Starting from the straightbladed configuration, 'a', shown in figure 1(a), the radial location of each blade section was displaced using a hyperbolic cosine distribution in order to yield the troposkien shape of configuration 'b', as shown in figure 1(b). Configuration 'c', shown in figure 1(c), was then obtained by applying helical blade twist around the rotor axis. The maximum radius, $R$, of each rotor is at the mid-span of the reference blade ('blade 1 ') of the turbine, and is identical for each of the three different configurations. This radius is used as the reference radius of the rotor when presenting non-dimensional data for the performance of the rotor. The tip speed ratio, $\lambda$, is defined as the ratio between the circumferential velocity at the mid-span of the blade, $\Omega R$, and the wind speed, $V_{\infty}$. The key parameters of the straight-bladed turbine, which is treated throughout this paper as the baseline configuration against which the other turbines are compared, are summarized in table 1 . The orientation of the blades and specification of the azimuth angle of the turbine with respect to the mid-span of blade 1 is shown in figure 2 .

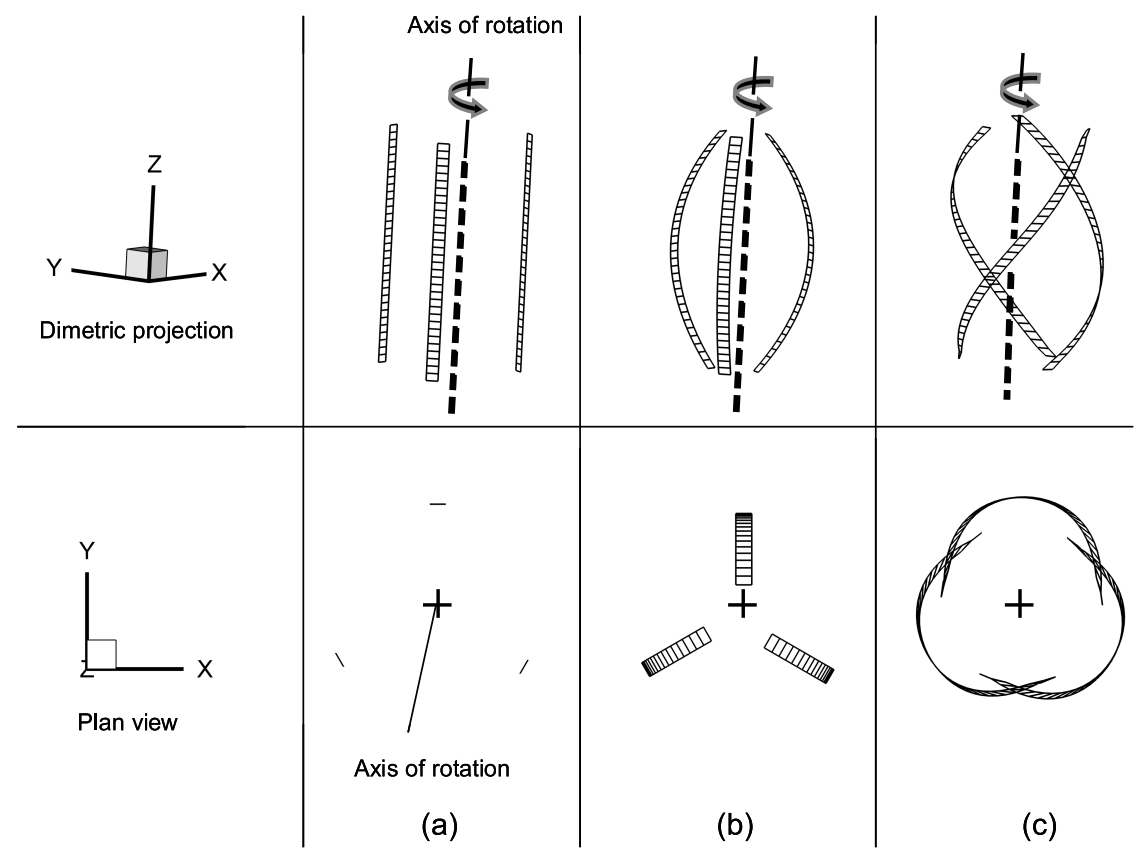

Figure 1. Geometry of the vertical-axis wind turbines with straight (a), curved (b), and helically twisted blades (c).

Table 1. Rotor parameters of the straight-bladed turbine.

Number of blades

Airfoil section

Rotational Reynolds number at blade mid-span

Chord-to-radius ratio at mid-span

Aspect ratio

Tip speed ratio 


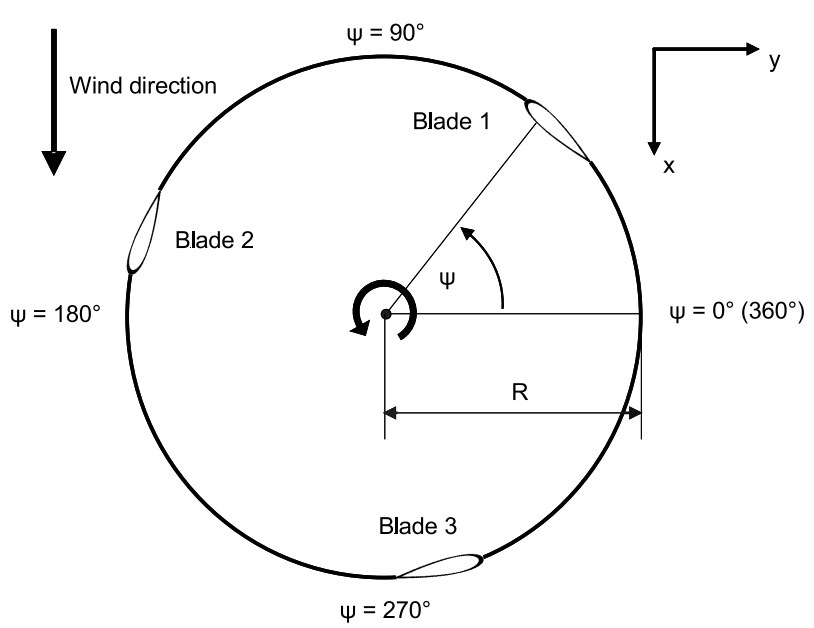

Figure 2. Diagram showing the wind direction, rotor azimuth definition and the relative positions of the rotor blades.

\section{Verification of Aerodynamic Predictions}

Before comparing the performance of the three different configurations of vertical-axis wind turbine that were defined in Section III, it is essential to establish first that the requisite fluid dynamic features within the flow surrounding the turbine rotor, and hence the aerodynamic loads on the blades of the rotor, are correctly simulated by the VTM. Figure 3 shows the VTM-predicted non-dimensional normal and tangential forces generated at the mid-span of a vertical-axis wind turbine with two straight blades, operating at a tip speed ratio of five, for which experimental measurements were obtained by Strickland, Smith and Sun. ${ }^{14}$

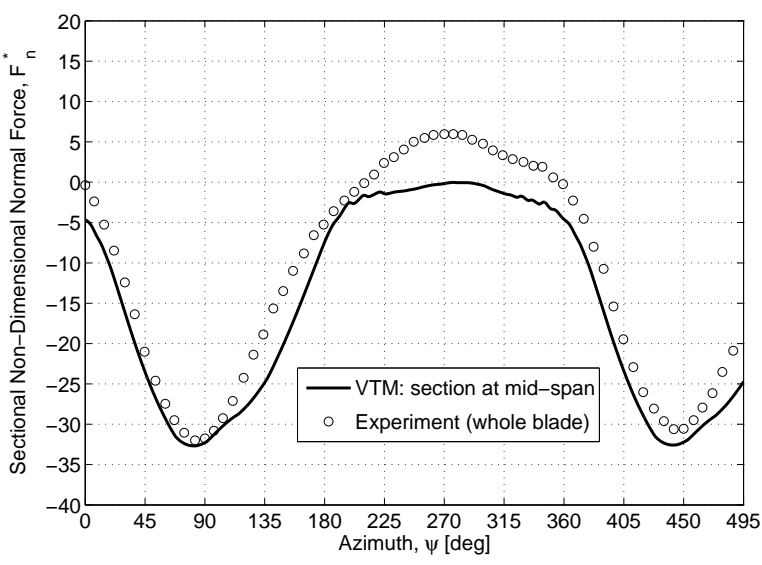

(a) Normal force

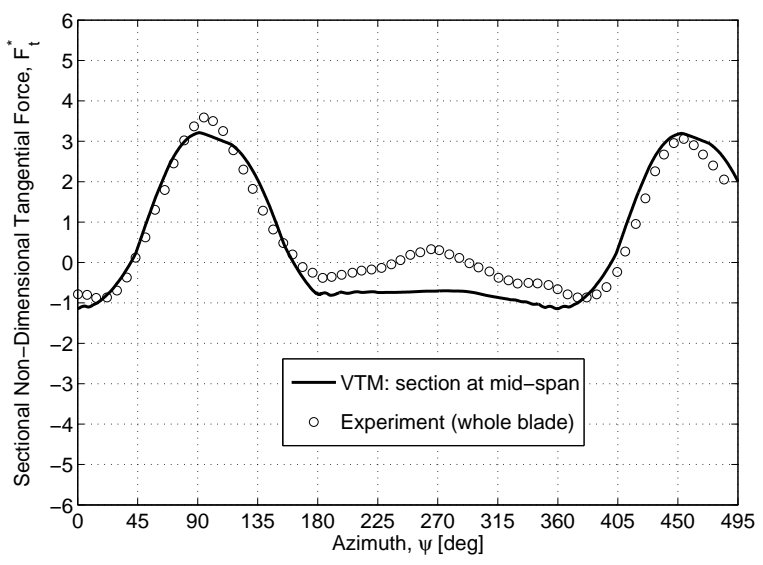

(b) Tangential force

Figure 3. Comparison of VTM-predicted non-dimensional forces with experimental measurements made by Strickland, Smith and Sun. ${ }^{14}$

The local blade velocity varies cyclically with azimuth due to the sinusoidal variation in the component of free stream velocity relative to the blade chord. On the windward side of the turbine, at $90^{\circ}$ azimuth, the free stream velocity vector is orthogonal to the blade chord, resulting in a high angle of attack and an associated peak in the blade loading. As the blade passes toward the leeward side of the turbine, between $180^{\circ}$ and $360^{\circ}$ azimuth, the flow that is induced by the wake of the turbine results in a reduction in the aerodynamic loading on the blades. In the upwind part of the revolution, between $0^{\circ}$ and $180^{\circ}$ azimuth, both the VTM-predicted non-dimensional normal force, shown in figure 3(a), and the non-dimensional tangential force, shown in figure 3(b), agree well with the experimental measurements. A discrepancy does occur between the VTM-predicted aerodynamic loading on the blades and the experimental measurements on the leeward side of the turbine, however. This is mainly due to the three-dimensional aerodynamic effects that are not captured accurately if only the mid-span section of the blade is considered. Downwind of the axis 
of rotation, each blade encounters its associated tip vortex and the tip vortices trailed from the other blade in previous revolutions. The interactions between the blade and the vortices in the wake generally occur close to the tip of the blade, whereas interactions with the tip vortices are almost completely absent at the mid-span of the blade, for the reasons described in greater detail in Section VI of this paper. A more detailed comparison between the VTM-predicted loading on this particular turbine and the equivalent experimental measurements performed by Strickland, Smith and Sun $^{14}$ is given by Scheurich, Fletcher and Brown. ${ }^{15}$

Overall, the VTM-predicted forces agree reasonably well with the experimental measurements, and the comparison provides confidence in the ability of the method to simulate accurately the aerodynamic loading on the blades of vertical-axis wind turbines.

\section{Cyclic Aerodynamic Loading}

The variation with azimuth of the aerodynamic loading on the blades of each of the three different vertical-axis wind turbine configurations features a cyclic component, which is described in detail in the present section, and an impulsive component which is examined in more detail in Section VI of this paper. The cyclic variation of the blade loading with azimuth is due to the sinusoidal variation of the component of free stream velocity with respect to the blade chord, and is characterized by a peak in the aerodynamic loading on the blade close to $90^{\circ}$ azimuth, i.e. in the upwind part of the rotor revolution. On the leeward side of the turbine, between $180^{\circ}$ and $360^{\circ}$ azimuth, however, the blades interact with the vortices in the wake of the turbine to produce a highly impulsive component to the loads on the system.

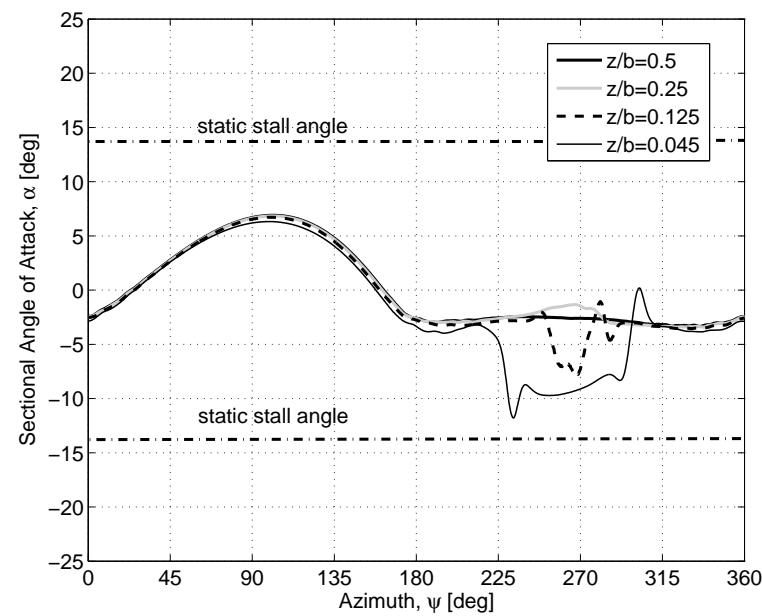

(a) Angle of attack

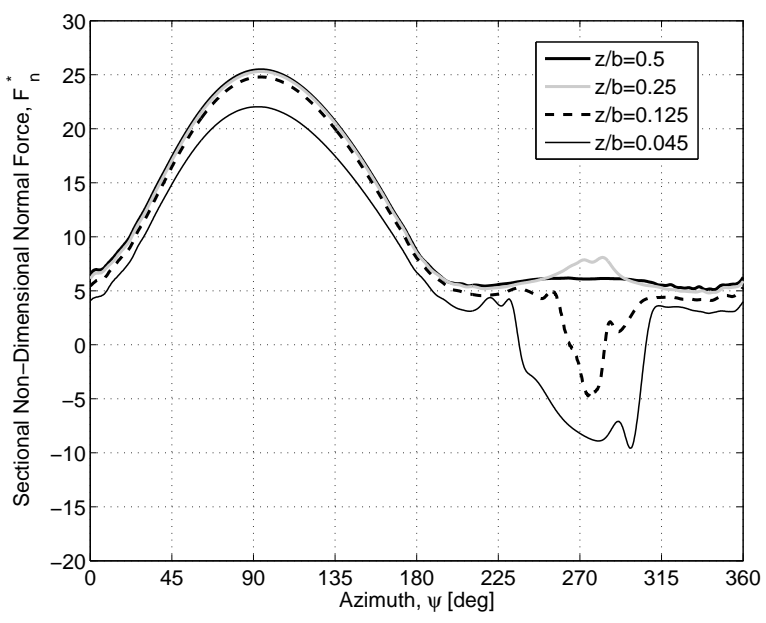

(c) Normal force

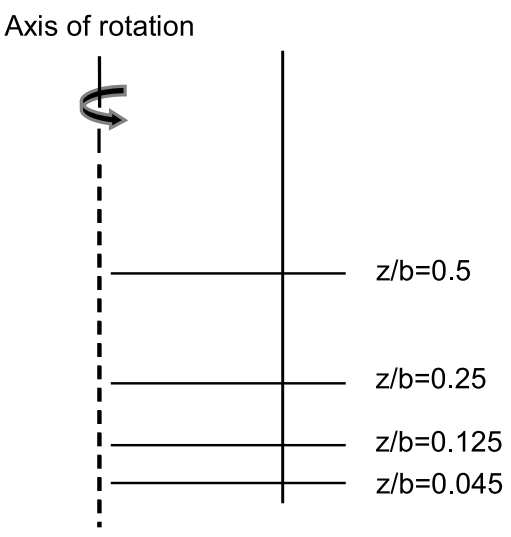

(b) Location

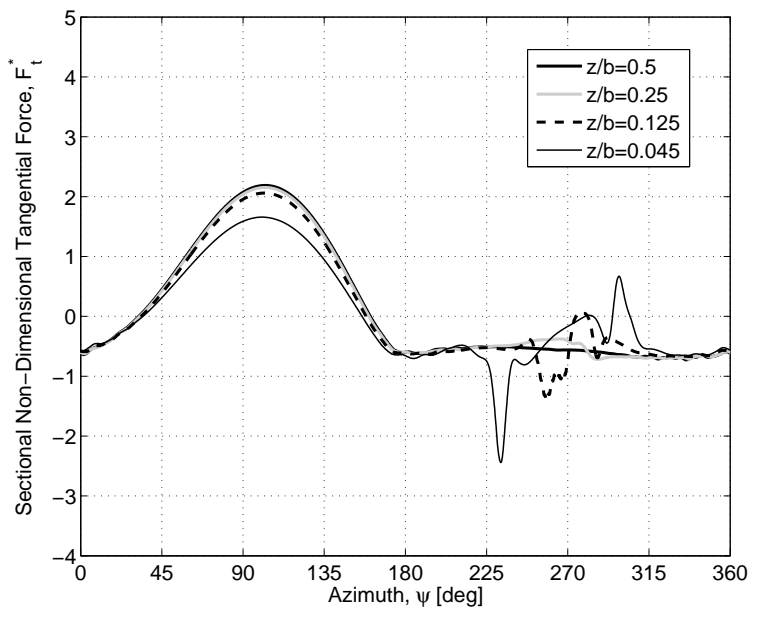

(d) Tangential force

Figure 4. VTM-predicted forces and angle of attack distribution over one complete rotor revolution at four different spanwise stations along the straight-bladed turbine (configuration ' $a$ '). 
Figure 4(a) shows the variation with azimuth of the angle of attack at four spanwise locations along the reference blade of the straight-bladed turbine, as defined in figure 4(b). The variation with azimuth of the sectional non-dimensional normal and tangential forces is presented in figures 4(c) and 4(d).

A peak occurs in the variation of the angle of attack, and consequently in the blade aerodynamic loading close to $90^{\circ}$ azimuth. The behavior of this turbine is thus very similar to that of the two-bladed turbine for which the blade loading was presented in figure 3. Figure 4(a) illustrates that the angle of attack close to the blade $\operatorname{tip}(z / b=0.125$ and $z / b=0.045)$ is lower than that nearer to the mid-span of the blade. This effect is caused by the finite span of the blade and thus the influence of the tip vortices in inducing a strong component of flow, normal to the blade chord, near the tips of the rotor blades. On the leeward side of the turbine, large, transient perturbations in the angle of attack are induced near to the tip of the blade. These impulsive perturbations are caused by interactions between the blade and the vortices that are trailed and shed from the blades in previous rotor revolutions, as discussed in more detail in Section VI of this paper.

Figure 5(a) shows the variation with azimuth of the angle of attack at four spanwise locations along the reference blade of the curved-bladed turbine, as defined in figure 5(b). The variation with azimuth of the sectional non-dimensional normal and tangential forces is presented in figures $5(\mathrm{c})$ and $5(\mathrm{~d})$.

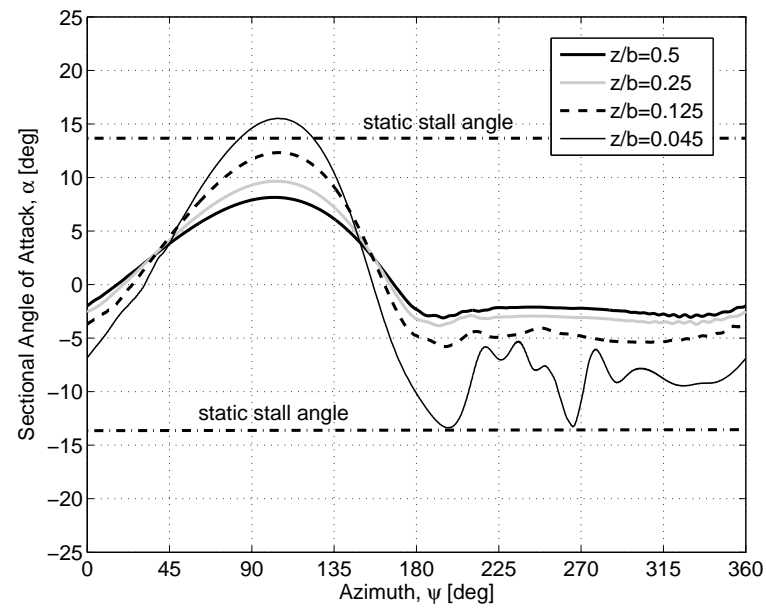

(a) Angle of attack

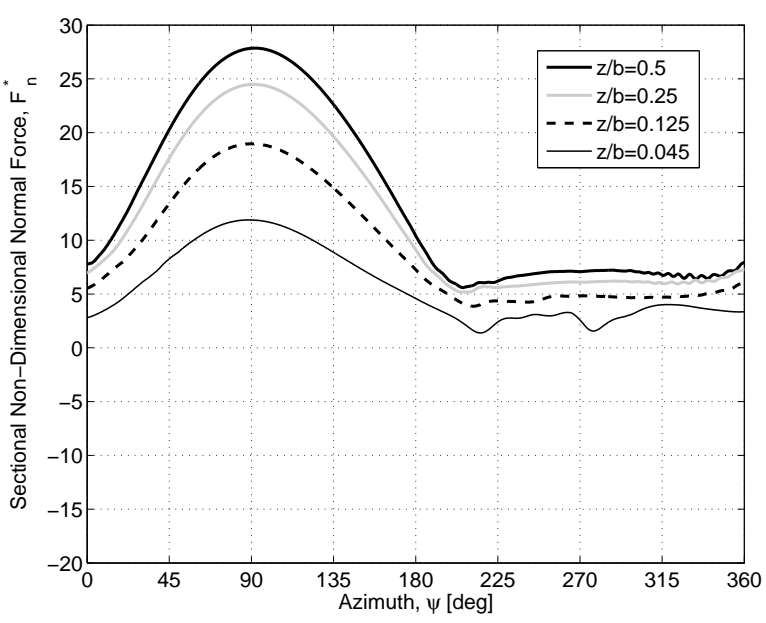

(c) Normal force

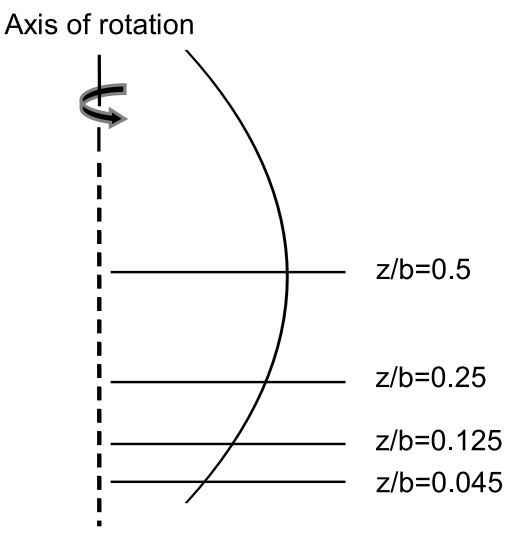

(b) Location

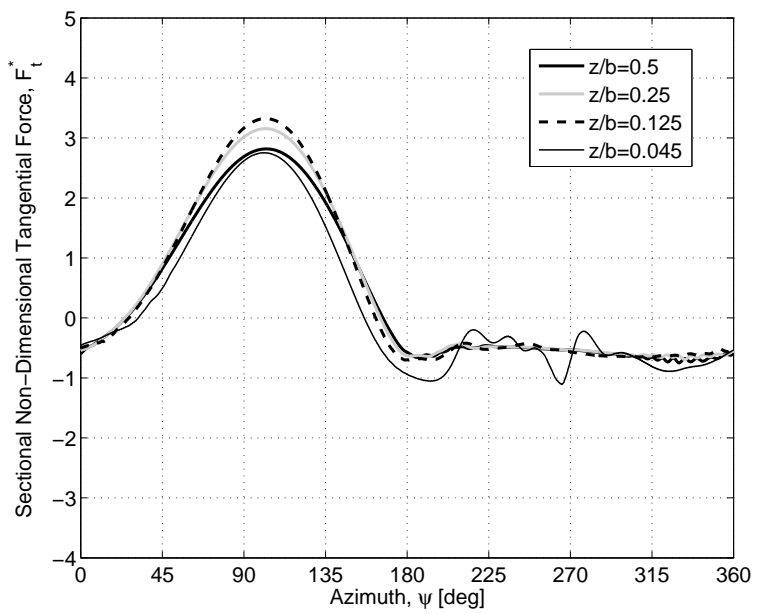

(d) Tangential force

Figure 5. VTM-predicted forces and angle of attack distribution over one complete rotor revolution at four different spanwise stations along the curved-bladed turbine (configuration ' $b$ '). 
Figure 5 shows the cyclic variation of the angle of attack with azimuth for the curved-bladed turbine to resemble that of the straight-bladed turbine, with a similar peak in the blade loading close to $90^{\circ}$ azimuth. The variation of the angle of attack along the blade span differs to that observed for the straight-bladed turbine, however, as the curvature of the blades results in a reduced radius, and, consequently, close to the blade tip, in a reduced circumferential component of velocity relative to the free stream. The amplitude of the oscillation of the local angle of attack close to the tip is thus significantly greater than that at the mid-span of the blade. Indeed, figure 5(a) shows that, near the tip of the blade, the angle of attack increases periodically beyond the static stall angle for the NACA 0015 airfoil. This indicates that the region near to the tips of the blades of the curved-bladed turbine configuration is subject to dynamic stall, even at moderate tip speed ratios. Figure 5 illustrates that the blades of this turbine are also subject to large, transient perturbations to the angle of attack, and thus to the blade loading, close to the tip of the blade when the blades pass through the wake that is produced by the turbine. These perturbations have a similar origin to those shown in figure 4 for the straight-bladed turbine but are confined to the most outboard elements of the blade.

Finally, figure 6(a) shows the variation with azimuth of the angle of attack at four spanwise locations along the reference blade of the turbine with helically twisted blades, as defined in figure 6(b). The variation with azimuth of the sectional non-dimensional normal and tangential forces is presented in figures $6(\mathrm{c})$ and $6(\mathrm{~d})$.

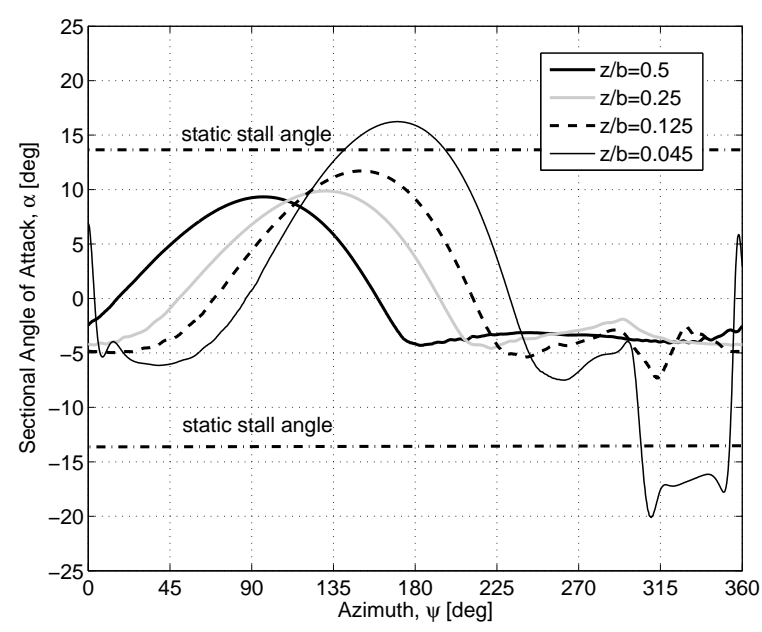

(a) Angle of attack

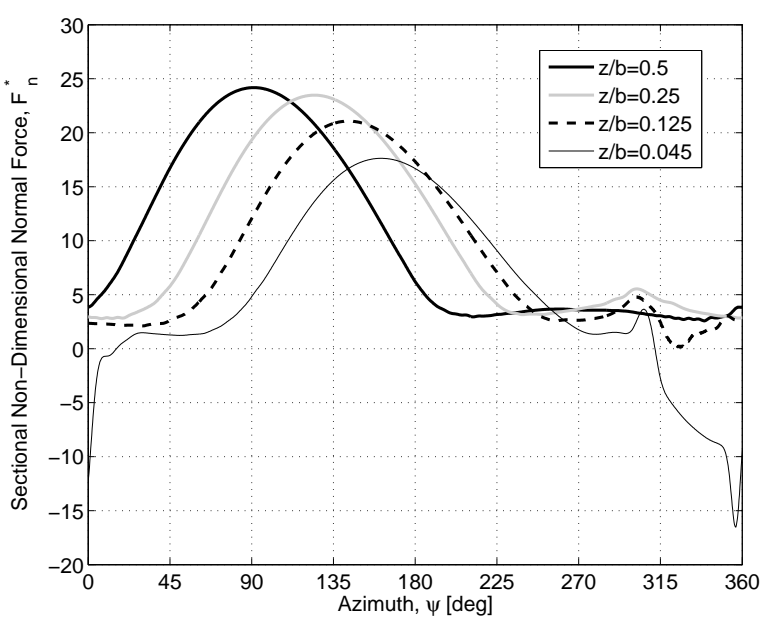

(c) Normal force

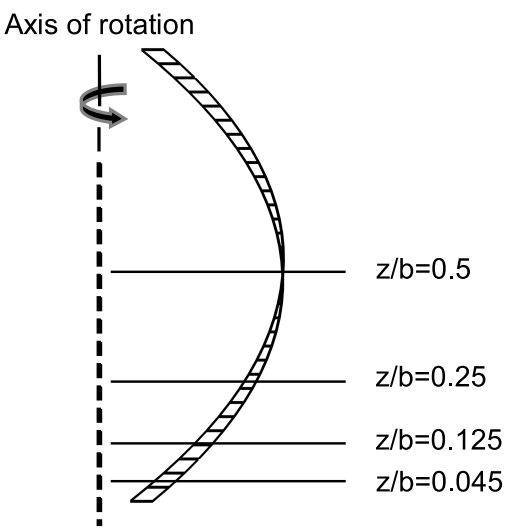

(b) Location

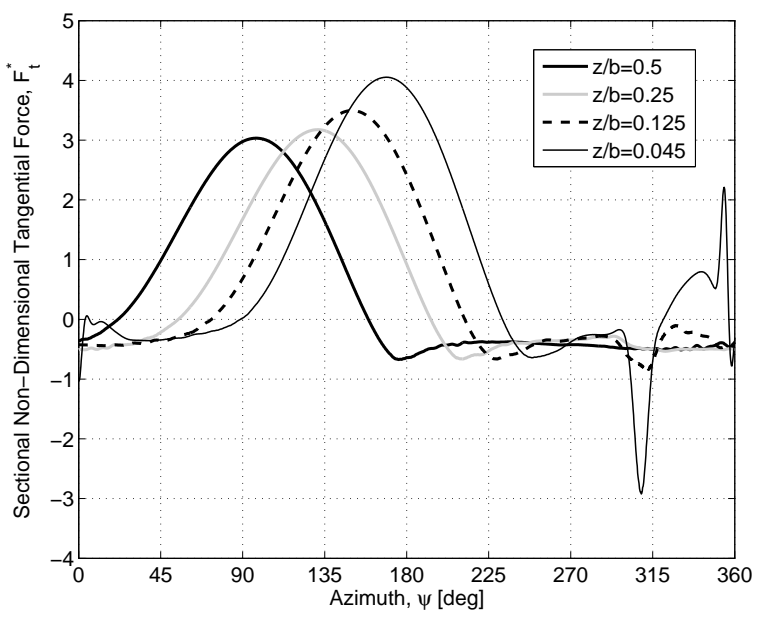

(d) Tangential force

Figure 6. VTM-predicted forces and angle of attack distribution over one complete rotor revolution at four different spanwise stations along the turbine with helically twisted blades (configuration 'c'). The azimuth $\psi$ is with respect to the section at the mid-span of the reference blade ('blade 1'). 
Figure 6(a) indicates that, near to its tips, the angle of attack of the reference blade of the turbine with helically twisted blades also periodically exceeds the static stall angle of the airfoil. This is, again, a consequence of the reduced circumferential component of velocity relative to the free stream near to the tips of the blades, which is associated with the reduced diameter of the rotor near the blade tips when compared to that at the mid-span of the blade. The prime consequence, however, of the helical twist that is applied to the turbine with configuration ' $c$ ' is the phase lag in the angle of attack and, thus, in the loading along the length of the blades. The progressive phase shift in the variation of the angle of attack, and consequently in the blade loading along the length of the blade is a result of the distribution of blade area over an azimuthal sector of the turbine, as seen in figure 1(c). In the downwind part of the revolution of the blade, between $180^{\circ}-360^{\circ}$ azimuth, impulsive perturbations in the variation of the angle of attack and the blade loading, although most marked near the tip of the blade, are also observed further inboard. This is indicative of a broader distribution of interactions between the blades and the vortices within the wake of the turbine with helically twisted blades than on the other two turbine configurations described in this paper. The sectional normal forces close to the blade tip are smaller than those experienced at the mid-span of the blade. Interestingly, the sectional tangential forces that are generated on the outboard part of the blade are slightly greater than the loads that are generated at the mid-span of the blade, however. The reduction in the local velocity that is experienced by the outboard portion of the blade that is due to its smaller radius might lead one to the conclusion that the sectional tangential force close to the blade tip should also be lower than that at mid-span of the blade. This effect is offset, however, by the higher angle of attack close to the tip of the blade which results in local tangential forces that are similar or even greater in magnitude than those experienced at the center of the blade. The importance of this observation is discussed further in Section VII of this paper, where the variation with azimuth of the torque that is produced by the different turbine configurations is compared.

\section{Impulsive Blade Loading due to Blade-Wake-Interaction}

The particular advantage of the Vorticity Transport Model is that it allows the wake of the turbine to be captured without significant spatial smearing of its structure during the many revolutions of the rotor in which the wake remains in close proximity to the blades. This renders the model particularly adept at resolving in detail the interaction between the blades of the turbine and the wake.

A three-dimensional representation of the wake that is produced by each of the three different turbine configurations is presented in figure 7. Each wake is visualized as a surface within the flow on which the vorticity has constant magnitude. Figures $7(\mathrm{a}), 7(\mathrm{c})$ and $7(\mathrm{e})$ illustrate the overall wake structure that is developed by each of the three turbine configurations, whilst, in order to reveal the complexity of the vortical structure within the wake, in figures $7(\mathrm{~b}), 7(\mathrm{~d})$ and $7(\mathrm{f})$ separate shades are used to show the vorticity that is created by each of the three blades of the turbines.

Figures 8(a), 8(b) and 8(c) show the vorticity distribution on a plane through the center of each of the three turbine configurations. This plane is oriented perpendicular to the ground and is aligned with the wind direction. The vorticity distribution is depicted at the instant of time when blade 1 is located at $270^{\circ}$ azimuth. The flow field is represented using contours of the component of vorticity perpendicular to the plane. The dark rendering corresponds to vorticity with a clockwise sense, and the light rendering to vorticity with a counter-clockwise sense of rotation. Figures 8(a) and 8(b) illustrate that the untwisted blades of the straight- and curved-bladed turbines result in a relatively symmetric distribution of vorticity in the wake downstream of the turbine. In contrast, the flow field of the turbine with helically twisted blades is characterized by a strong asymmetry in the distribution of vorticity between the upper and lower halves of the turbine, as is shown in figure $8(\mathrm{c})$. The visualization of the wake allows the origins to be identified of the localized impulsive perturbations that were observed in the variation of the angle of attack and the aerodynamic loading on the blades of each of the three turbine configurations. Indeed, the large, transient perturbations in the variation of the angle of attack and the blade loading of the straight-bladed turbine that were observed in figure 4 can be related directly to the interaction between the reference blade and a region of vorticity in the wake, as depicted in figure 8(a). This region of vorticity consists of the vortices that were trailed and shed from the blades in previous rotor revolutions. It can also be seen why, in the absence of significant interactions with the tip vortices, the angle of attack, and consequently the blade loading, at the mid-span of the blade $(z / b=0.5)$ does not exhibit the same impulsive perturbations that are observed at blade sections further outboard. This observation is important as it implies that an appropriate threedimensional representation of the wake is an essential component of any model that is to capture accurately the aerodynamics of vertical-axis wind turbines.

9 of 16 


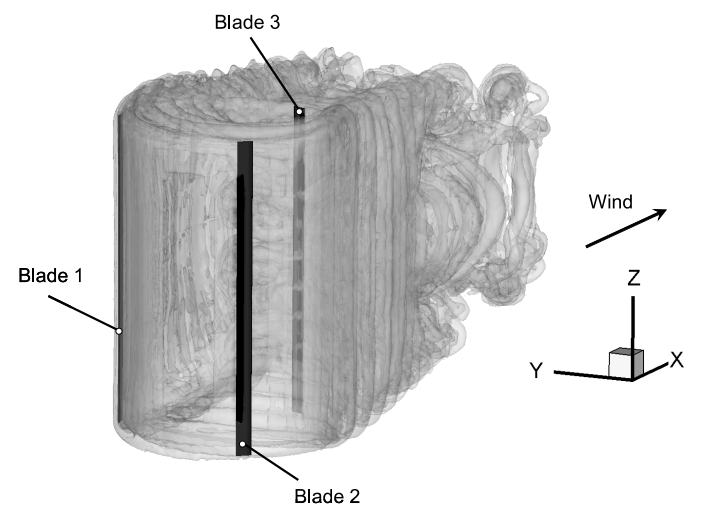

(a)
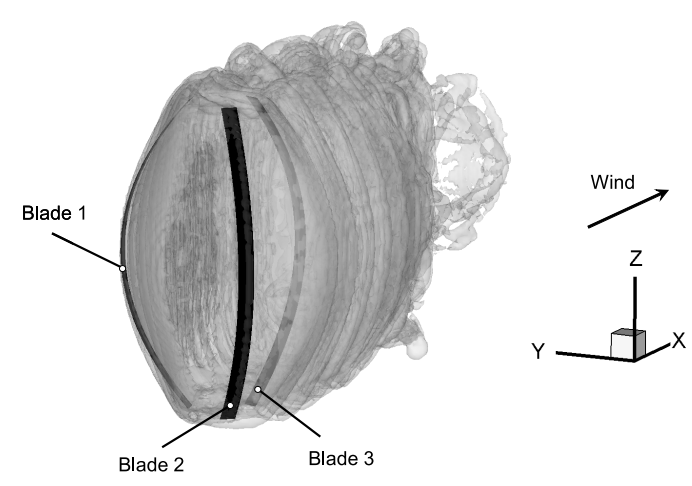

(c)

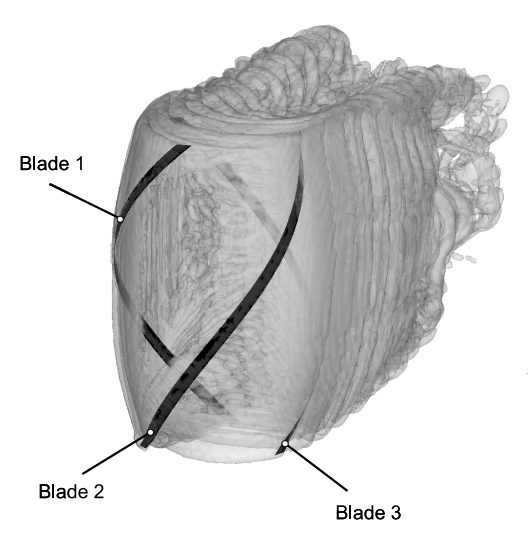

(e)

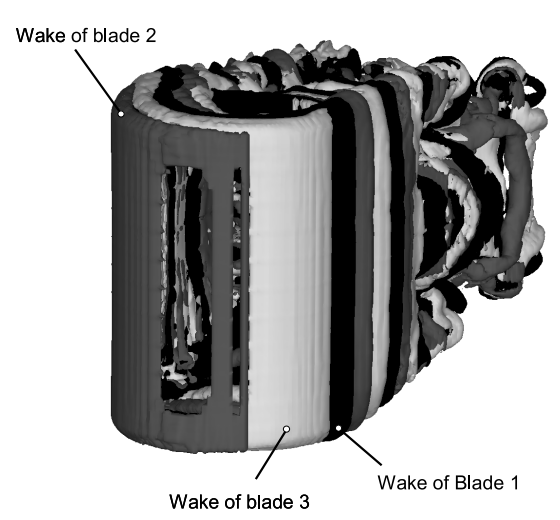

(b)

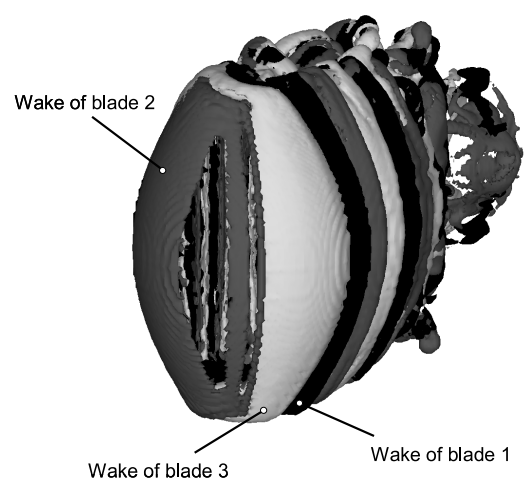

(d)

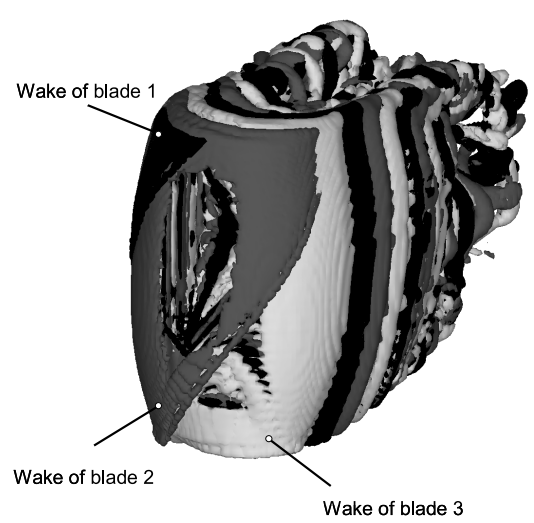

(f)

Figure 7. Three-dimensional graphical representation of the computed flow fields surrounding the turbine configurations with straight blades (a,b), curved blades (c,d), and helically twisted blades (e,f). The wake geometry is visualized by rendering a surface on which the vorticity has constant magnitude. The sub-figures on the left show the entire vorticity field that is developed by the turbine, whereas the subfigures on the right show the vorticity that is developed by each individual blade. 


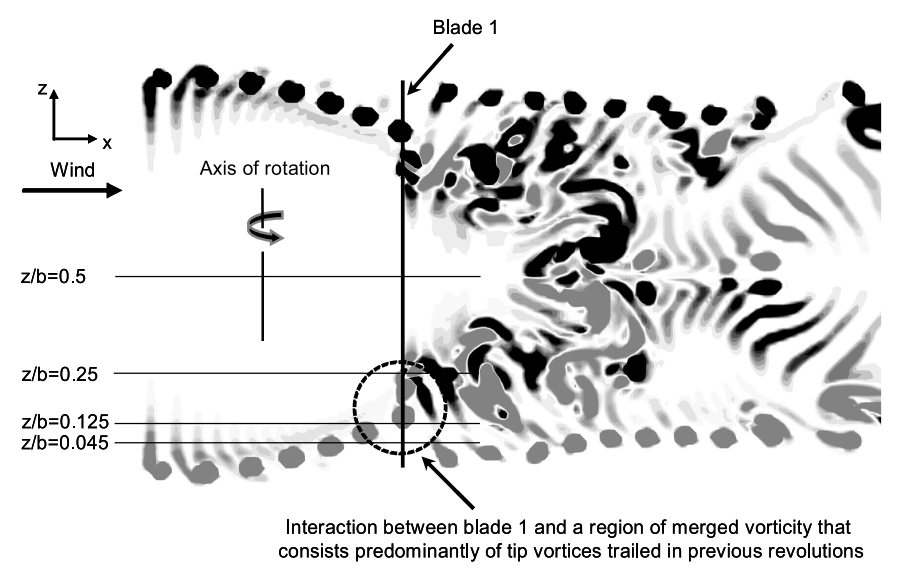

(a) straight-bladed turbine

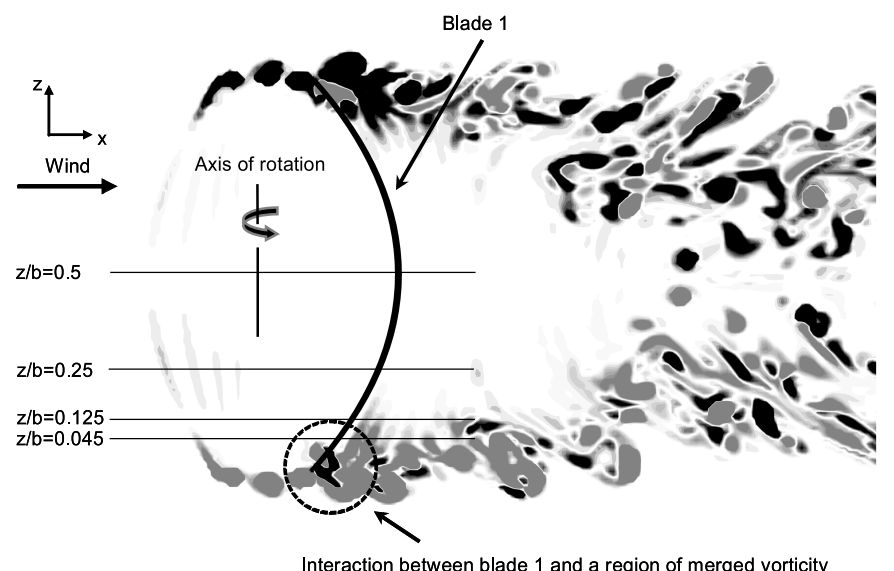

(b) curved - bladed turbine

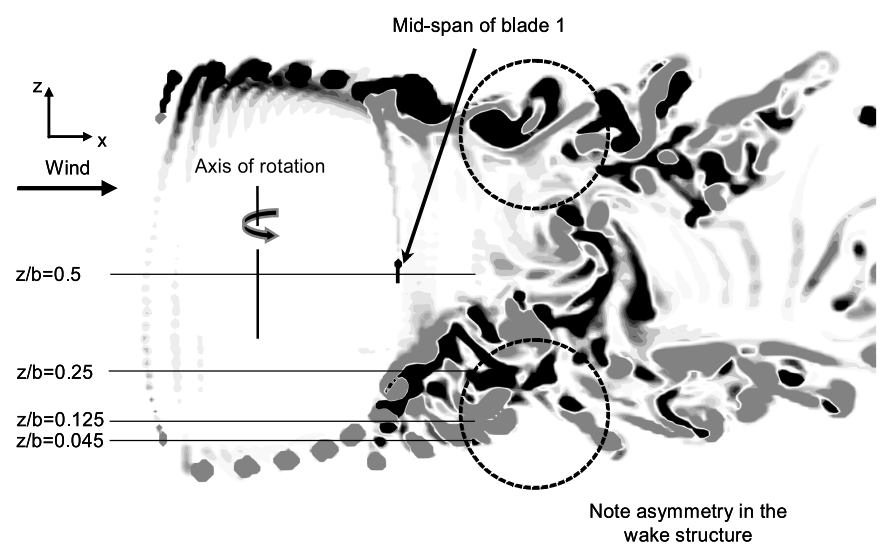

(c) turbine with helically twisted blades

Figure 8. Computed vorticity fields surrounding the three turbine configurations that were investigated, represented using contours of vorticity on a vertical plane that contains the axis of rotation of the turbine, and that is aligned with the wind direction. Blade 1 is located at azimuth $\psi=270^{\circ}$. 


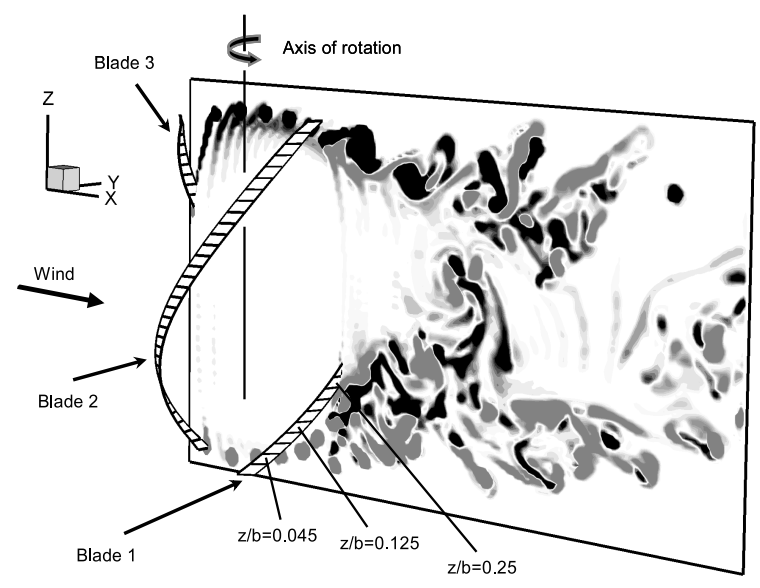

(a) mid - span of blade 1 at $\psi=300^{\circ}$

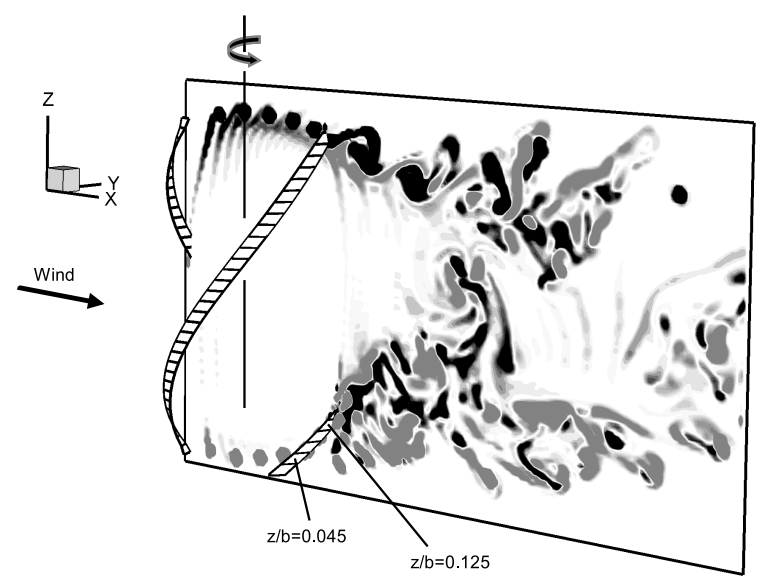

(b) mid - span of blade 1 at $\psi=320^{\circ}$

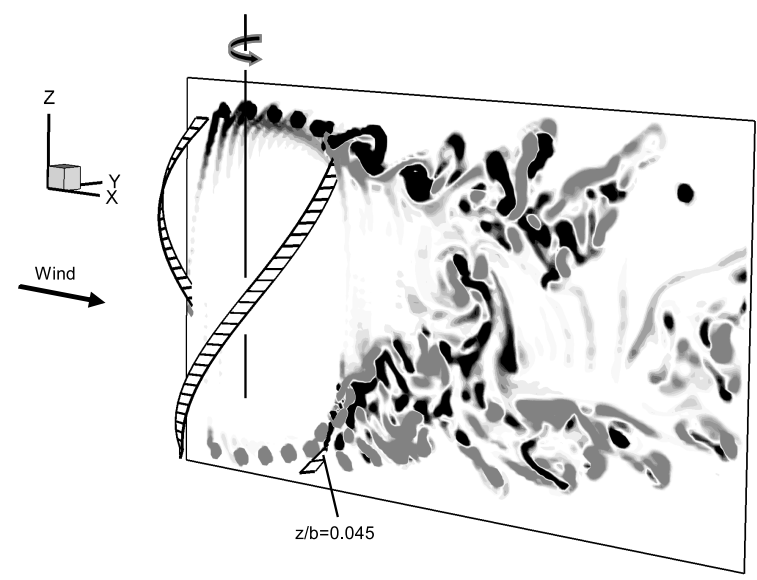

(c) mid - span of blade 1 at $\psi=340^{\circ}$

Figure 9. Computed vorticity field surrounding the turbine with helically twisted blades. Visualization by contours of the vorticity component that is perpendicular to a vertical plane that contains the axis of rotation of the turbine, and that is aligned with the wind direction. 
The vorticity distribution that is produced in the wake of the turbine with curved blades is shown in figure 8(b). Compared to the extent of the blade-wake interactions that occur in the straight-bladed turbine system, the blade-wake interactions within the curved-bladed turbine system can be seen to be confined to a smaller portion of the blade near to the tip. This observation is consistent with figure 5 which showed the transient perturbations in the angle of attack and the aerodynamic loading to be confined to a much smaller region near to the very tip of the blade than was the case with the straight-bladed turbine.

The distribution of vorticity in the wake that is produced by the turbine with helically twisted blades is shown in figure 8(c), and is reproduced again in figure 9 with the rotor in three different azimuth orientations to expose the migration along the length of the blade, as the turbine rotates, of the regions of most intense blade-vortex interaction. In figures $9(\mathrm{a}), 9(\mathrm{~b})$ and $9(\mathrm{c})$, the mid-span of the reference blade is located at $300^{\circ}, 320^{\circ}$ and $340^{\circ}$ azimuth, respectively. At $300^{\circ}$ azimuth, principal interactions with the vortices within the wake occur at section $z / b=0.25$ of blade 1 , but as the turbine rotates, these interactions move outboard along the blade. This migration is the origin of the broad distribution along the span of the blades of the impulsive perturbations in the variation of the angle of attack and the blade loading that were observed in figure 6 .

\section{Variation in Torque and Power Coefficient}

Figure 10 shows the contribution to the overall torque from the four spanwise locations of the reference

blade of each turbine configuration, as defined in figures 4(b), 5(b) and 6(b). The angle of attack, and consequently the local tangential force coefficient, for those sections close to the tip of the blades of the turbine with helically twisted blades is higher when compared with those sections nearer to the mid-span of the blade. This can be understood once it is realized that blade curvature results in a smaller radius for the sections that are located close to the blade tip in comparison to those that are located toward the mid-span of the blade. The reduced radius of the sections close to the blade tip also results in a lower local velocity at those sections, however. Indeed, it is the combination of a lower local velocity and a higher force coefficient due to a higher angle of attack that results in a relatively uniform distribution of torque being developed along the length of the blades of the helically twisted turbine, as shown in figure 10(c).

The azimuthal variation of the loading on the blades yields significant unsteadiness in the torque and hence the power that is produced by a vertical-axis wind turbine if its blades are not twisted. The introduction of helical blade twist can reduce significantly the oscillations in power output from the turbine. This is demonstrated in figure 11, where the variation of the unsteady component of the power coefficient, $\Delta C_{P}$, for one turbine revolution is presented for the three different turbine configurations. Although the three different wind turbines have identical key rotor parameters, the difference in their aerodynamic design leads, naturally, to different absolute values of their power coefficients even if they are operated at the same tip speed ratio. In order to make a rigorous comparison between the turbine configurations, the variation of the power coefficient from the mean power coefficient is compared in figure 11. It should be noted that at the tip speed ratio of five at which the simulations were conducted, the mean power coefficients for the turbines with straight, curved and helically twisted blades were $0.094,0.235$ and 0.386 , respectively.

The straight- and curved-bladed turbines exhibit significant unsteadiness in power coefficient over the course of a single turbine revolution. The oscillations in the power coefficients of the straight- and curvedbladed turbines have three coherent peaks close to $90^{\circ}, 210^{\circ}$ and $330^{\circ}$ azimuth; this reflects the location of the peak in the variation of the torque of each single blade close to $90^{\circ}$ azimuth, as shown in figures 10 (a) and 10(b). The power coefficient of the turbine with helically twisted blades, in contrast, is relatively steady. Despite each blade section of the helically twisted blade experiencing fluctuations in blade loading with azimuth, the more even distribution of its blade area around the azimuth of the turbine results in a significant reduction in the unsteadiness of the torque that is transmitted through the shaft, and consequently in a reduction in the unsteadiness of the power coefficient that it produces, when compared to the turbines with untwisted blades. The azimuthal variation of the power coefficient of the turbine with helically twisted blades is not entirely smooth, however. A residual unsteadiness in the power output of the turbine results partially from the incomplete overlap of the three blades, but also from the perturbations to the blade loading that are caused by the blade-vortex interactions shown in Section VI of this paper. 


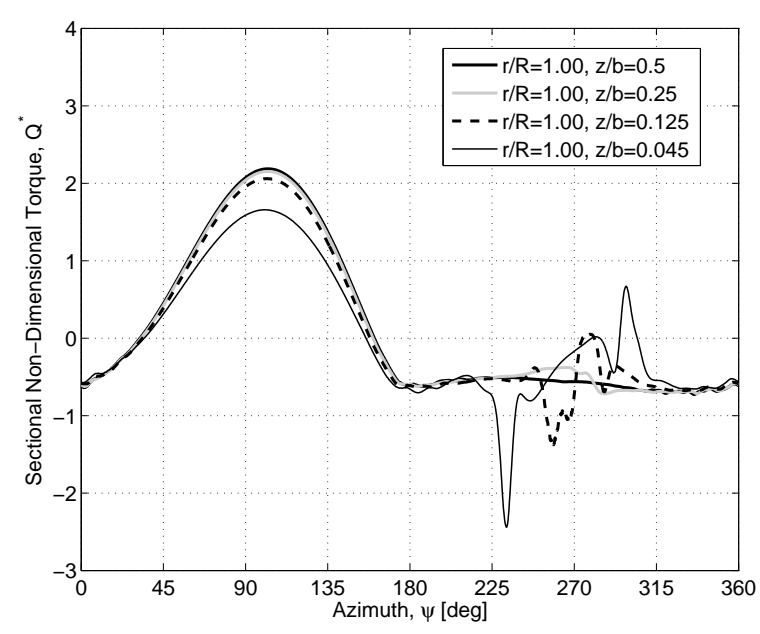

(a) straight - bladed turbine

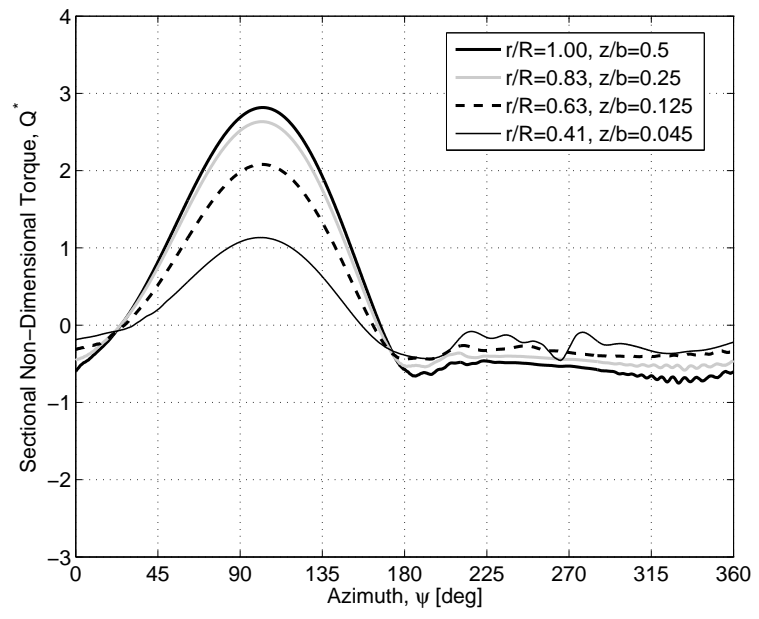

(b) curved - bladed turbine

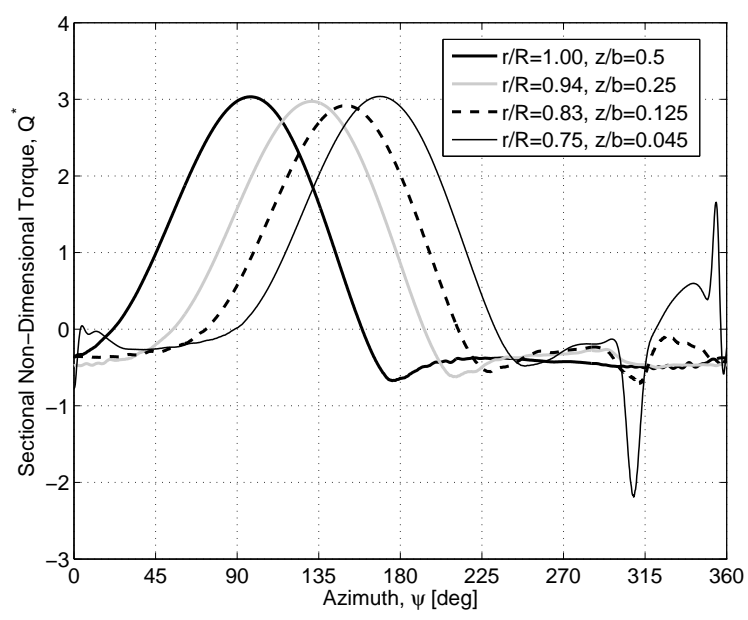

(c) turbine with helically twisted blades

Figure 10. VTM-predicted sectional non-dimensional torque at four blade sections for the three turbine configurations that were investigated. 


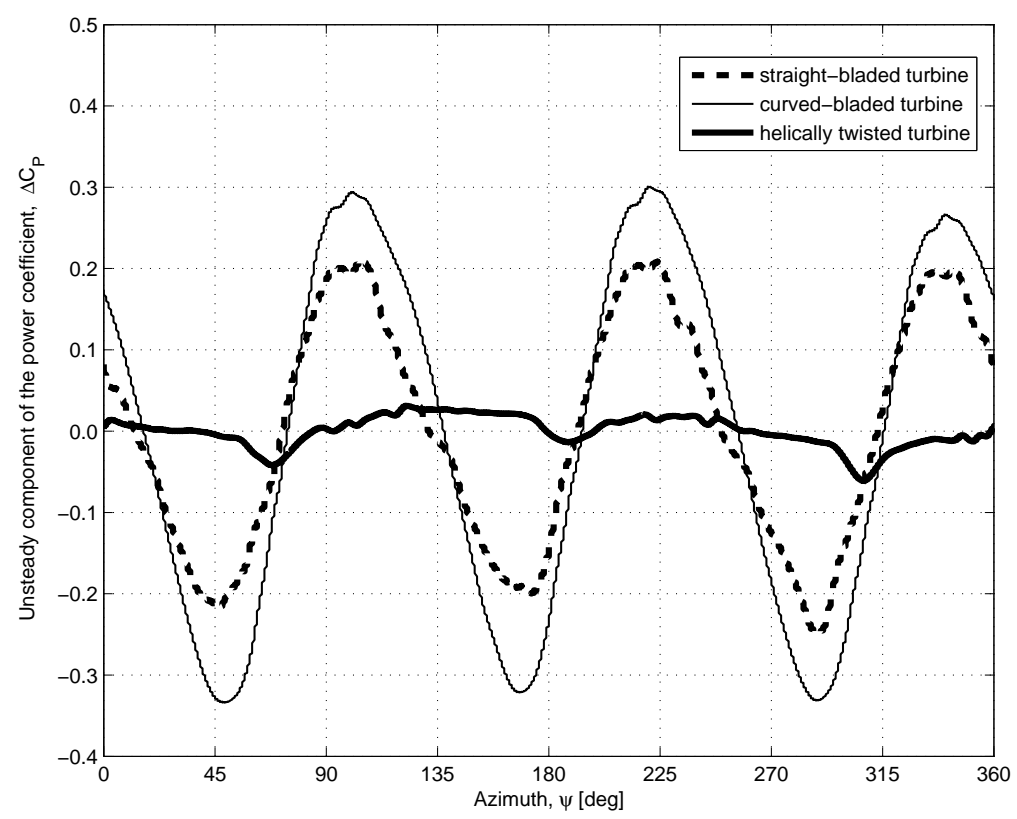

Figure 11. VTM-predicted variation of power coefficient with azimuth angle for the three turbine configurations that were investigated.

\section{Conclusion}

The aerodynamic performance and wake dynamics of three vertical-axis wind turbines, one with straight blades, another with curved blades and another with a helically twisted blade configuration, have been investigated using the Vorticity Transport Model (VTM). The VTM explicitly conserves the vorticity within the flow field surrounding the turbine, thus enabling the influence on turbine performance of the structure and evolution of the vortical wake that is induced by the rotor to be analyzed in detail. Vertical-axis wind turbines with either straight or curved blades are known to suffer from blade loads that vary considerably with azimuth; this results in a power output that contains a substantial oscillatory component at the blade passage frequency of the turbine. The variations in the blade loading can fatigue the rotor structure and reduce the design life of the turbine. By comparison, a turbine with helically twisted blades yields a relatively steady power output. Despite the helically twisted blades still individually experiencing oscillations in blade loading with azimuth, a relatively steady force distribution for the entire turbine is achieved through an almost uniform distribution of blade area around the azimuth. The power output of the turbine with helically twisted blades is not entirely smooth, however, as the mutual interactions between the blades and the vortices within the wake that is developed by the rotor do still result in localized impulsive perturbations to the aerodynamic loading on the blades.

Blade curvature results in a reduced circumferential velocity relative to the free stream velocity at the outboard region of the blade when compared to that at the mid-span of the blade. This is because the effective radius of the blade sections is smaller near to the blade tips than at the mid-span of the blade. The outboard regions of the blades of the curved-bladed configuration therefore experience oscillations in angle of attack that are of larger amplitude than those at the mid-span of the blade. The angle of attack can exceed, at least transiently, the range in which the lift coefficient varies linearly with angle of attack, and can thus manifest as dynamic stall at lower tip speed ratios than for the straight-bladed configuration.

The analysis that is presented in this paper reveals that an appropriate model for the three-dimensional wake that is produced by a vertical-axis wind turbine is essential in order to capture the interactions between the blades of the turbine and the vortices in the wake, and thus, that such a model is a crucial component of any numerical scheme that is to predict accurately the aerodynamic performance of vertical-axis wind turbines. 


\section{References}

${ }^{1}$ Mertens, S., "Wind Energy in the Built Environment," Multi-Science Publishing Co. Ltd, Brentwood, UK, 2006.

${ }^{2}$ Darrieus, G. J. M., "Turbine Having its Rotating Shaft Transverse to the Flow of the Current," US Patent 1,835,018, December 1931.

${ }^{3}$ Klimas, P. C., "Darrieus Rotor Aerodynamics," Transactions of the ASME. Journal of Solar Energy Engineering, Vol. 104, 1982, pp. 102-105.

${ }^{4}$ Brown, R. E., "Rotor Wake Modelling for Flight Dynamic Simulation of Helicopters," AIAA Journal, Vol. 38, No. 1, 2000, pp. 57-63.

${ }^{5}$ Brown, R. E., and Line, A. J., "Efficient High-Resolution Wake Modelling Using the Vortex Transport Equation," AIAA Journal, Vol. 43, No. 7, 2005, pp. 1434-1443.

${ }^{6}$ Toro, E., "A Weighted Average Flux Method for Hyperbolic Conservation Laws," Proceedings of the Royal Society of London, Series A: Mathematical and Physical Sciences, Vol. 423, No. 1864, 1989, pp. 401-418.

${ }^{7}$ Leishman, J. G., and Beddoes, T. S., "A Semi-Empirical Model for Dynamic Stall," Journal of the American Helicopter Society, Vol. 34, No. 3, 1989, pp. 3-17.

${ }^{8}$ Gupta, S., and Leishman, J. G., "Dynamic Stall Modelling of the S809 Aerofoil and Comparison with Experiments," Wind Energy, Vol. 9, No. 6, 2006, pp. 521-547.

${ }^{9}$ Beddoes, T. S., "A Third Generation Model for Unsteady Aerodynamics and Dynamic Stall," Westland Helicopters Ltd., RP-908, 1993.

${ }^{10}$ Niven, A. J., and Galbraith, R. A. McD., "Modelling Dynamic Stall Vortex Inception at Low Mach Numbers," Aeronautical Journal, Vol. 101, No. 1002, 1997, pp. 67-76.

${ }^{11}$ Hand, M. M., Simms, D. A., Fingersh, L. J., Jager, D. W., Cotrell, J. R., Schreck, S., Larwood, S. M., "Unsteady Aerodynamics Experiment Phase VI:Wind Tunnel Test Configurations and Available Data Campaigns," National Renewable Energy Laboratory (NREL), USA, NREL TP-500-29955, 2001.

${ }^{12}$ Fletcher, T. M., Brown, R. E., Kim, D. H., and Kwon, O. J., "Predicting Wind Turbine Blade Loads using Vorticity Transport and RANS Methodologies," European Wind Energy Conference, Marseille, France, 16-19th March 2009.

${ }^{13}$ Fletcher, T. M., and Brown, R. E., "Simulating Wind Turbine Interactions using the Vorticity Transport Equations," 28th ASME Wind Energy Symposium, Orlando, Florida, USA, 5-8th January 2009.

${ }^{14}$ Strickland, J. H., Smith, T., and Sun, K., "A Vortex Model of the Darrieus Turbine: An Analytical and Experimental Study," Sandia National Laboratories, USA, SAND81-7017, June 1981.

${ }^{15}$ Scheurich, F., Fletcher, T. M., and Brown, R. E., "Simulating the Aerodynamic Performance and Wake Dynamics of a Vertical-Axis Wind Turbine," Wind Energy, manuscript under review, September 2009. 